\title{
Chemotherapy-Related Nausea and/or Vomiting
}

National Cancer Institute

\section{Source}

National Cancer Institute. Chemotherapy-Related Nausea and/or Vomiting. NCI

Thesaurus. Code C107528.

Nausea and/or vomiting co-occurring with a cycle of chemotherapy. 